\title{
Community Treatment of Severe, Refractory Obsessive-Compulsive Disorder.
}

\author{
Mark J. Boschen* \\ School of Psychology and Griffith Health Institute, Griffith University, Gold Coast, \\ Australia
}

Lynne M. Drummond

St George's, University of London, and OCD/BDD Services, South West London and St George's Mental Health NHS Trust, London, United Kingdom.

\footnotetext{
* Corresponding Author: Dr Mark Boschen, School of Psychology, Griffith University, Parklands Drive, Southport Q 4215, Australia. Email: m.boschen@griffith.edu.au, Ph: +61 755528283.
}

\section{Author Note}

We wish to thank Ms Anusha Govinda and Mr Bartholomew Tenerowicz for their assistance in collating and compiling the original data. 


\begin{abstract}
Despite the existence of effective psychological and pharmacological interventions for obsessive-compulsive disorder (OCD), there are a large proportion of individuals for whom intervention is not effective. The study reports on the results of a community treatment service for individuals with treatment-refractory OCD who have not benefitted from previous cognitive-behavioural or pharmacological treatment by community mental health services. A total of 205 individuals accepted for treatment by a specialist community OCD treatment service in London were provided with a combination of behavioural, cognitive, and pharmacological treatment within a specialist OCD service, with 158 completing treatment. Treatment was associated with significant reduction in clinician-rated and self-reported OCD symptoms after 12 weeks, with further reductions in OCD symptoms over a subsequent 12 week period. A significant reduction in symptoms of depression was also observed after 12 and 24 weeks. Approximately $40 \%$ of individuals treated experienced clinically significant improvement in their symptoms, with approximately $10 \%$ deemed to have recovered by the end of treatment. Our results add to previous research into refractory OCD, and provide support for the existence of specialist community treatment services which may provide assistance to individuals who have not responded to previous treatment.
\end{abstract}

KEYWORDS: Obsessive-Compulsive Disorder, Behaviour Therapy, CognitiveBehaviour Therapy. 


\section{Community Treatment of Severe, Refractory Obsessive-Compulsive Disorder.}

Obsessive-compulsive disorder is a serious psychiatric disorder in which individuals experience repeated intrusive thoughts, impulses, or imagery as well as repeated overt and covert behavioural acts aimed at reducing anxiety or discomfort (APA, 2000). Lifetime prevalence rates have been estimated at approximately $1.3 \%$, with 12 month prevalence rates of $0.54 \%$ (Somers, Goldner, Waraich, \& Hsu, 2006). Individuals with OCD experience functional impairment (Markarian et al., 2010), as well as reduced quality of life (Fontenelle et al., 2010). Research into OCD has been steadily growing over recent decades (Boschen, 2008).

The effectiveness of psychological and pharmacological intervention for OCD has been established in several previous meta-analyses (e.g., Abramowitz, 1997; Cox, Swinson, Morrison, \& Lee, 1993; Eddy, Dutra, Bradley, \& Westen, 2004; RosaAlcázar, Sánchez-Meca, Gómez-Conesa, \& Marín-Martínez, 2008, van Balkom et al., 1994). Effective treatment is also associated with improvements in quality of life (Moritz et al., 2005; Norberg, Calamari, Cohen, \& Riemann, 2008). Despite the existence of effective treatments, however, there are a large proportion of individuals who do not respond to treatment, along with an even larger proportion (>70\%) who do not reach an asymptomatic state after intervention (Fisher \& Wells, 2005). Ascertaining who will benefit most from treatment is also difficult (Boschen, Drummond, Pillay, \& Morton, 2010; Farrell \& Boschen, 2011; Stewart, Yen, Stack, \& Jenike, 2006)

Some individuals with OCD experience a level of symptom severity that requires intervention by specialist inpatient or community treatment teams. There have, however, been relatively few scientific reports of treatment outcome in this 
most severely affected population. The earliest analysis of treatment outcome in severe OCD was reported by Drummond in 1993. In this study, the author reported on an inpatient cohort of 49 individuals with treatment-refractory OCD. The treatment approach strongly emphasised the use of behaviour therapy, and was associated with clinical improvement in the majority $(63.3 \%)$ of patients. Similar successful findings have been reported in the treatment other severe OCD cohorts (Stewart, Stack, Farrell, Pauls, \& Janike, 2005; Thornicroft, Colson, \& Marks, 1991). Community treatment of a cohort of 62 individuals with severe, treatmentrefractory OCD, was reported by Boschen, Drummond, and Pillay in 2008. In this study, the authors provided outcome results for individuals referred to the Trustwide tertiary service of South West London and St George's Mental Health NHS Trust between 2006 and 2007. In this study, treatment involved an integrated multidisciplinary approach including behavioural, cognitive, and pharmacological interventions, with a distinct focus on the use of exposure with response prevention (ERP) techniques. In this group, overall OCD symptom severity declined significantly over a 12 and 24 week treatment period. On average, individuals' symptoms reduced by $21.7 \%$ in the first week, and a further $11.5 \%$ in the subsequent 12 week period. The uncontrolled effect size of the intervention $(d=1.22)$ was comparable to previous meta-analyses in less severe patients. Depression symptoms also reduced significantly during treatment. Examination of treatment outcome for each individual in the cohort demonstrated that $40 \%$ showed a statistically reliable reduction in OCD symptom severity, while $19 \%$ showed clinically significant improvement, and only 5\% were deemed to have recovered from their OCD.

We sought to evaluate treatment outcome in a larger cohort of individuals treated as outpatients by our specialist OCD treatment service since the original 
published study by Boschen et al. in 2008. In this prospective study, we examined the effectiveness of treatment for severe OCD involving a combination of psychological and pharmacological interventions at both the group, and individual level. Similarly to Boschen et al. (2008), we also examined individual change scores. We also benchmarked our results against those reported previously in meta-analyses of OCD treatment, and the previous outpatient outcomes in severe OCD by Boschen et al. (2008)

\section{Method}

\section{Participants}

Participants were drawn from referrals to the Trustwide tertiary referral service of the South West London and St George's Mental Health NHS Trust. The service has a catchment area which covers the five London boroughs of Kingston, Merton, Richmond, Sutton, and Wandsworth, and a total adult population of approximately one million. A total of 266 individuals were referred for treatment over the period January 2008 to August, 2011, of which 205 met inclusion criteria and were accepted for treatment. All individuals had their diagnosis of OCD confirmed by a consultant psychiatrist with expertise in OCD. To meet inclusion criteria, all individuals were required to have had an unsatisfactory response to cognitive behavioural therapy provided by their local community mental health team. Most individuals had also been previously prescribed serotonergic medication, and some had also received augmentation of this medication with dopamine antagonists, again with unsatisfactory response as judged by their community mental health team. Details of the previous treatment experience of the cohort are presented in Table 1. The only additional exclusion criterion was that patients did not have a primary 
psychotic illness. Further details of the service have been previously described by Drummond et al. (2008).

\section{Materials}

Outcome was assessed using a range of psychometrically validated measures. All of these have been previously used to assess outcome in treatment of OCD, and also in outcome for severe, treatment-resistant OCD (Boschen et al., 2008). OCD symptom severity was assessed using the clinician-rated Yale-Brown ObsessiveCompulsive Scale (Y-BOCS; Goodman, Price, Rasmussen, Mazure, Delgado, et al., 1989; Goodman, Price, Rasmussed, Mazure, Fleischmann, et al., 1989), and the selfreport Padua Inventory (Sanavio, 1988). The Y-BOCS was administered by a psychiatrist, or other specialist mental health worker with experience in the assessment and treatment of OCD. Depression symptoms were assessed using the self-report Beck Depression Inventory (BDI; Beck, 1993). The measures were administered after 12 and 24 weeks as part of routine clinical assessment in all participants.

\section{Procedure}

Upon referral, cases were discussed by the multidisciplinary treating team which includes psychiatrists, cognitive behavioural therapists, and other allied health practitioners, and treatment plans are generated. Interventions within the service were ideographic rather than manualised, and were tailored to the individual needs of each patient. Treatment usually consisted of a combination of behavioural, cognitive, and pharmacological interventions, with a distinct emphasis on the use of behavioural methods such as exposure and voluntary response prevention. All staff had specialist 
expertise in the treatment of OCD. Most treatments took place in the community, either in the patients home or in the local community.

Patients were treated in the community with cognitive behavioural therapy focussing on graded exposure and self-imposed response prevention. In the cohort, $78 \%$ of patients completed at least 24 hours of psychological therapy with the treating team. Sessions varied in length and duration depending on the individual needs and characteristics of the patient. For example, some individuals required home-based therapy, involving three to four hours at home in any one day, to ensure full exposure and response prevention, while others received hourly sessions at the clinic. All sessions were conducted individually, and homework tasks were generated after each intervention session in collaboration with the patient.

The treating team consisted of a consultant psychiatrist, and other therapists who were all accredited with the British Association for Behavioural and Cognitive Therapy. The consultant psychiatrist provided advice and supervision during treatment. All therapists had extensive experience with treatment of OCD and had been working with refractory $\mathrm{OCD} / \mathrm{BDD}$ for a minimum of five years.

As the overwhelming majority of patients were already prescribed appropriate medication prior to admission, very few changes were made to pharmacological treatment. An SSRI antidepressant was added in $4.0 \%$ of cases, while a dopamine antagonist was added in $2.0 \%$.

\section{Results}

A total of 205 patients were accepted for treatment into the service. Of these patients for whom there was an intention to treat, $158(77.1 \%)$ completed treatment (see Figure 1). When patients either did not complete treatment, or there was missing 
data, the last observation was carried forward to yield a conservative estimate of change during the treatment period. Of this final intent-to-treat sample of 205 individuals, 94 (45.9\%) were male, 109 (53.2\%) were female, and one did not have their sex recorded. Mean age at intake was 38.48 years $(S D=12.01)$. The mean age of OCD onset was 19.03 years $(S D=9.55)$, while patients had a mean duration of illness of 19.57 years $(S D=12.44)$. Demographic characteristics such as marital, employment, and accommodation status are presented in Table 2.

All patients had a primary diagnosis of OCD. Almost half of the 205 patients accepted for treatment had at least one additional psychiatric diagnosis, with the most common comorbid condition being major depressive disorder, occurring in approximately one third of the sample. Comorbid conditions are presented in Table 3.

The mean total Y-BOCS score for the sample at the pre-treatment baseline was 26.32 This is at the higher end of the range of initial Y-BOCS scores reported for five OCD treatment outcome studies by Fisher \& Wells (2005), and comparable to the severe OCD outpatient treatment baseline severity reported by Boschen et al. (2008). This, combined with the requirement that previous treatment had been unsuccessful, supports the assertion that our sample was one with severe and refractory OCD prior to treatment.

\section{Symptom Change during Treatment}

There was a significant reduction in clinician-rated severity of obsessivecompulsive disorder symptoms, as measured by the Y-BOCS, over the course of treatment $\left(F_{(2,404)}=193.73, p<.001\right)$. Comparison between pre-treatment and 12 weeks after commencement of treatment demonstrated a significant reduction of $18.5 \%$ in symptoms over this time $\left(M_{\mathrm{Pre}}=26.32, S D_{\mathrm{Pre}}=5.30, M_{12 \mathrm{Wk}}=21.46, S D_{12 \mathrm{Wk}}\right.$ 
$=7.14,95 \% C I_{\text {Diff }}=4.05$ to $\left.5.66, t=11.89, d f=202, p<.001\right)$. There was further improvement shown by the patient cohort over the following 12 week period also $\left(M_{24 \mathrm{Wk}}=17.41, S D_{24 \mathrm{Wk}}=8.13,95 \% C I_{\text {Diff }}=3.26\right.$ to $4.84, t=10.12, d f=202, p<$ $.001,33.9 \%$ reduction from baseline). Change in Y-BOCS scores are shown in Figure 1.

Similar results were seen when self-reported OCD symptoms, as measured by the Padua Inventory, were used as a measure of outcome. There was significant change over the course of the treatment period $\left(F_{(2,294)}=44.64, p<.001\right)$. Selfreported symptoms declined between intake and 12 week assessments $\left(M_{\mathrm{Pre}}=81.68\right.$, $S D_{\text {Pre }}=38.91, M_{12 \mathrm{Wk}}=71.42, S D_{12 \mathrm{Wk}}=39.67,95 \% C I_{\text {Diff }}=6.63$ to $13.88, t=5.59, d f$ $=147, p<.001,12.6 \%$ reduction from baseline), as well as between 12 week and 24 week assessments $\left(M_{24 \mathrm{Wk}}=62.70, S D_{24 \mathrm{Wk}}=39.11,95 \% C I_{\text {Diff }}=4.96\right.$ to $11.39, t=$ 5.03, $d f=152, p<001 ; 23.2 \%$ reduction from baseline). The reduction in Padua Inventory total scores is shown in Figure 2.

Self-reported depression symptoms, as measured by the BDI also showed a reduction across treatment $\left(F_{(2,356)}=80.09, p<.001\right)$. There was a significant decline in depression symptoms between intake and 12 weeks $\left(M_{\text {Pre }}=24.65, S D_{\text {Pre }}=11.45\right.$, $M_{12 \mathrm{Wk}}=19.74, S D_{12 \mathrm{Wk}}=11.59,95 \% C I_{\text {Diff }}=3.81$ to $6.00, t=8.82, d f=178, p<.001$, $19.9 \%$ reduction from baseline), as well as a further reduction in depression symptoms between 12 and 24 weeks of treatment $\left(M_{24 \mathrm{Wk}}=17.57, S D_{24 \mathrm{Wk}}=11.04\right.$, $95 \% C I_{\text {Diff }}=1.20$ to $2.96, t=4.66, d f=180, p<.001,28.7 \%$ reduction from baseline). Figure 1 shows the reduction in depression symptoms during treatment.

\section{Individual Change}


Individual patient scores on the Y-BOCS were used to evaluate whether individual change had occurred for each participant. Reliable change was deemed to have occurred if total Y-BOCS scores reduced by at least 10 points during treatment (Boschen et al., 2008; Fisher \& Wells, 2005; Jacobsen \& Truax, 1991). Patients were deemed to have clinically improved if their final Y-BOCS score was closer to that of the normal population mean than the clinical OCD population mean (i.e., $\leq 14$ points; Fisher \& Wells, 2005). Patients were judged to have recovered if their final Y-BOCS score was no higher than seven points, as recommended by Fisher \& Wells (2005).

Of the 203 individuals for which data was available, $86(42.4 \%)$ demonstrated reliable change on the Y-BOCS, with all of these ending with fewer symptoms at the end of treatment compared to intake. A total of 81 individuals (39.9\%) had improved over the course of treatment, while $21(10.3 \%)$ were judged to have recovered.

Alternative criteria for evaluating outcome have also been proposed by Pallanti et al. (2002). According to these criteria, 25 individuals (12.2\%) were classified as partial responders (i.e., reduction of between $25 \%$ and $35 \%$ on the YBOCS), while an additional 100 (48.8\%) were categorised as full responders (i.e., $>35 \%$ reduction in Y-BOCS scores). A total of 91 (44.4\%) were classified as in remission (i.e., final Y-BOCS scores < 16), while $21(10.2 \%)$ were classified as in recovery (i.e., final Y-BOCS score $<8$ ).

\section{Benchmarking Against Previous Research}

The results from our community treatment were compared against two relevant previous studies. A meta-analysis by Eddy, Dutra, Bradley, and Westen (2004) was chosen as this provided uncontrolled effect sizes for pharmacological and psychological interventions. As can be seen in Figure 4, the uncontrolled effect size 
in the current study of $d=1.30$ is comparable to effect sizes seen in the previous meta-analysis of OCD treatment, as well as the previous research in a similar earlier cohort reported by Boschen et al. in 2008 .

\section{Predictors of Treatment Response}

We also examined a number of variables to assess whether these were associated with change in OCD symptoms during treatment. Initial visual inspection of scatter plots did not suggest the presence of any non-linear bivariate relationships. Change in OCD symptoms was not significantly correlated with age at intake $(r=.04$, $p=.61)$, age of onset of $\mathrm{OCD}(r=.01, p=.90)$, or duration of illness $(r=.04, p=$ .65). There was no different in improvement between those in a current relationship versus those who were single, separated or divorced $(t=0.60, d f=170, p=.55)$. Nor was there a difference between those living alone versus those living with a partner, parents or supported accommodation $(t=0.05, d f=107, p=.96)$. Individuals in fulltime employment showed a significantly greater percentage reduction in OCD scores during treatment, compared with those not in full-time employment $\left(M_{\text {Fulltime }}=\right.$ $\left.40.26 \%, M_{\text {NotFulltime }}=26.81 \%, t=3.49, d f=185, p=.001\right)$. Severity of OCD symptoms $(r=.05, p=.48)$ and depression symptoms $(r=-.10, p=.18)$ at intake were also unrelated to improvement. ${ }^{1}$

The presence of comorbid psychiatric conditions had an inconsistent impact on outcome. Presence of a comorbid major depressive disorder was not related to reduction in OCD symptoms $(t=1.38, d f=201, p=.17)$. In contrast, the presence of any comorbid psychiatric disorder was associated with changes in OCD symptoms $(t$ $=2.42, d f=201, p=.02$ ), where those with an additional diagnosis performed more poorly than those without $\left(M_{\text {Comorbid }}=37.95 \%, M_{\text {NoComorbid }}=28.85 \%\right)$. 


\section{Discussion}

This study examined the effectiveness of a community-based, multidisciplinary, ideographic, multimodal intervention for treatment-resistant OCD. It provided evidence suggesting that specialist treatment of OCD may provide significant therapeutic effect, even for individuals who have not responded to previous psychological and pharmacological treatment. Significant reduction in OCD symptoms was observed after 12 weeks of treatment, with additional reduction in symptoms after a further 12 weeks. Severity of depressive symptoms also reduced over the course of treatment. When data from individual patients was examined approximately $40 \%$ showed clinically significant improvement, while $10 \%$ were judged to have recovered during the treatment period. The effect size calculated for our intervention was similar to that observed in a similar cohort by Boschen et al. (2008), and also similar to the effect size reported in previous meta-analyses of psychological and pharmacological treatment of OCD (Eddy et al., 2004). When a range of demographic and clinical measures were examined for their effect on outcome, only employment status and the presence of other comorbid diagnoses showed any relationship to reduction in OCD symptoms.

Our results are comparable to those reported by Boschen et al. (2008) who examined an earlier, smaller cohort of individuals treated within the same service. As mentioned above, the effect size of 1.30 observed in the current study was similar to the effect size of 1.22 reported by Boschen et al. Individuals in the current study showed greater rates of statistically reliable improvement (42\% vs $40 \%$ ), clinically significant improvement (40\% vs 19\%), and recovery (10\% vs 5\%), compared with Boschen et al. (2008). As the cohorts were drawn from the same catchment areas 
using the same inclusion criteria, and were treated by the same team of clinicians, this improvement in outcome is difficult to conclusively explain, but may reflect an increasing level of expertise and refinement of treatment technique within the service. Alternatively, the current analysis had considerably less missing data than the previous analysis of Boschen et al., and as such required less use of last observations, and as such may be a more realistic, less conservative assessment of the change that occurred in the cohort.

Our results are also in accordance with other previous research into treatmentresistant OCD interventions. Other authors who have examined treatment of severe, treatment resistant OCD (e.g., Drummond, 1993; Stewart et al., 2005; Thornicroft et al., 1991) have also demonstrated that specialist treatment interventions can be successful when applied to individuals who have not responded to previous treatment. More broadly, our results also support previous research that attests to the effectiveness of pharmacological and psychological approaches to the treatment of OCD (e.g., Eddy et al., 2004; Rosa-Alcázar et al., 2008).

We believe that our results carry important implications for the treatment of individuals with OCD. It is known that many individuals treated both pharmacologically and using cognitive and behavioural treatments may not respond to initial treatment within a general community mental health model. Our results demonstrate that a specialist intervention service, with staff who have extensive experience and expertise in the treatment of $\mathrm{OCD}$, can provide symptom reduction for many who have not responded to earlier intervention. As a result it supports the existence of tertiary referral services aimed at assisting those with treatment-resistant OCD who have not responded to less intensive or less specialised interventions. 
The reader should be mindful of several limitations in the current research when interpreting our findings. First, our study did not have a control group for comparison against those treated within our service. As our service is primarily a clinical service, rather than a research-based unit, having a control group has not been possible. We believe, however, that our results are important despite the lack of a control group for comparison. All individuals referred to our service had longstanding OCD that had not responded to previous intervention. As such, it is reasonable to assume that spontaneous symptom reduction was likely to be rare, and as such our findings are most likely due to the specific intervention used, rather than other non-specific factors. Second, the multimodal nature of our treatment, involving psychological and pharmacological interventions delivered simultaneously, makes it difficult to ascertain the specific effective components of the treatment, or to elucidate the specific mechanisms of change that led to reduced OCD symptoms. Treatment was provided in an individually tailored, formulation-driven manner, and as such was not standardised. There were also no checks of treatment integrity, beyond clinical supervision and clinical case conferences where cases were discussed with the treating team. Third, our data collection did not include details about the specific content of obsessions and compulsive behaviours for each participant. This limits our ability to more fully describe our sample, and also prevents examination of the effectiveness of the treatment for different symptoms.

We believe that there is considerable need for further research into OCD, and specifically into treatment for those individuals with the most severe, treatmentresistant forms of the condition. Firstly, there is an obvious need to continue to develop treatment methods that may assist a greater number of individuals with OCD. More than half of our cohort did not demonstrate clinically meaningful reduction in 
their OCD symptoms as a result of treatment. These individuals, with a particularly refractory variant of the condition, do not seem to be helped by any of the currently accepted evidence-based treatments for OCD. Development of new or refined psychological and pharmacological treatments may be required to offer benefit to these more severely affected patients. Secondly, further research is also needed into variables which predict treatment response or non-response in severe OCD. Identification of such variables would allos for the identification of people who are most likely to benefit from specialist treatment services. Thirdly, it is important that future research examine the underlying determinants of treatment resistance so that these can be addressed in future treatment. Where specific cognitive, behavioural, or biological mechanisms of treatment resistance can be identified, this would provide direction for clinicians and researchers aiming to address these as a way of increasing the probability of treatment success.

\section{Conclusion}

The current study presented an investigation of multimodal community treatment for individuals with severe, treatment refractory OCD. Our results provided preliminary evidence that specialist outpatient services may provide assistance to a substantial proportion of individuals for whom no previous psychological or pharmacological treatment has been effective. 


\section{References}

Abramowitz, J.S. (1997). Effectiveness of psychological and pharmacological treatments for obsessive-compulsive disorder: A quantitative review. Journal of Consulting and Clinical Psychology, 65, 44-52.

American Psychiatric Association (2000). Diagnostic and Statistical Manual of Mental Disorders (4th ed. text rev.). Washington DC: American Psychiatric Press.

Beck, A.T. (1993). Beck Depression Inventory Manual. San Antonio: The Psychological Corporation.

Boschen, M.J. (2008). Publication trends in individual anxiety disorders: 1980-2015. Journal of Anxiety Disorders, 22, 570-575.

Boschen, M.J., Drummond, L.M., \& Pillay, A. (2008). Treatment of severe, treatment refractory obsessive-compulsive disorder: A study of inpatient and community treatment. CNS Spectrums, 13, 1056-1065.

Boschen, M.J., Drummond, L.M., Pillay, A., \& Morton, K. (2010). Predicting outcome of treatment for severe, treatment resistant OCD in inpatient and community settings. Journal of Behavior Therapy and Experimental Psychiatry, 41, 90-95.

Cottraux, J., Note, I., Yao, S.N., Lafont, S., Note, B., Mollard, E., Bouvard, M., Sauteraud, A., Bourgeois, M., \& Dartigues, J.F. (2001). A randomized controlled trial of cognitive therapy versus intensive behavior therapy in obsessive-compulsive disorder. Psychotherapy \& Psychosomatics, 70, 288297.

Cox, B.J., Swinson, R.P., Morrison, B., Lee, P.S. (1993). Clomipramine, fluoxetine, and behavior therapy in the treatment of obsessive-compulsive disorder: A 
meta-analysis. Journal of Behavior Therapy and Experimental Psychiatry, 24, 149-153.

Drummond, L.M. (1993). The treatment of severe, chronic, resistant obsessivecompulsive disorder: An evaluation of an in-patient programme using behavioural psychotherapy in combination with other treatments. British Journal of Psychiatry, 163, 223-229.

Drummond, L.M., Pillay, A., Kolb, P., et al. (2008). The introduction of a community model for the treatment of obsessive-compulsive disorder and body dysmorphic disorders. Psychiatry Bulletin, 32, 336-340.

Eddy, K.T., Dutra, L., Bradley, R., \& Westen, D. (2004). A multidimensional metaanalysis of psychotherapy and pharmacotherapy for obsessive-compulsive disorder. Clinical Psychology Review, 24, 1011-1030.

Farrell, L.J., \& Boschen, M.J. (2011). Treatment outcome in adult OCD: Predictors and processes of change. Asia Pacific Journal of Counselling and Psychotherapy, 2, 82-97.

Fisher, P.L., \& Wells, A. (2005). How effective are cognitive and behavioural treatments for obsessive-compulsive disorder? Behaviour Research and Therapy, 43, 1543-1558.

Fontenelle, I.S., Fontenelle, L.F., Borges, M.C., Prazeres, A.M., Rangé, B.P., Mendlowicz, M.V., \& Versiani, M. (2010). Quality of life and symptom dimensions of patients with obsessive-compulsive disorder. Psychiatry Research, 179, 198-203.

Franklin, M.E., Abramowitz, J.S., Kozak, M.J., Levitt, J.T., \& Foa, E.B. (2000). Effectiveness of exposure and ritual prevention for obsessive-compulsive 
disorder: randomized compared with nonrandomized samples. Journal of Consulting and Clinical Psychology, 68, 594-602.

Goodman, W.K., Price, L.H., Rasmussen, S.A., Mazure, C., Delgado, P., Heninger, G.R., \& Charney, D.S. (1989). The Yale-Brown Obsessive Compulsive Scale: II. Validity. Archives of General Psychiatry, 46, 1012-1016.

Goodman, W.K., Price, L.H., Rasmussen, S.A., Mazure, C., Fleischmann, R.L., Hill, C.L., Heninger, G.R., \& Charney, D.S. (1989). The Yale-Brown Obsessive Compulsive Scale: I. Development, use, and reliability. Archives of General Psychiatry, 46, 1006-1011.

Jacobsen, N.S., \& Truax, P. (1991). Clinical significance: A statistical approach to defining meaningful change in psychotherapy research. Journal of Consulting and Clinical Psychology, 66, 136-142.

Lindsay, M., Crino, R., \& Andrews, G. (1997). Controlled trial of exposure and response prevention in obsessive-compulsive disorder. British Journal of Psychiatry, 171, 135-139.

Markarian, Y., Larson, M.J., Aldea, M.A., Baldwin, S.A., Good, D., Berkeljon, A., Murphy, T.K., Storch, EA., \& McKay, D. (2010). Multiple pathways to functional impairment in obsessive-compulsive disorder. Clinical Psychology Review, 30, 78-88.

McLean, P.D., Whittal, M.L., Thordarson, D.S., Taylor, S., Sochting, I., Koch, W.J., Paterson, R., \& Anderson, K.W. (2001). Cognitive versus behavior therapy in the group treatment of obsessive-compulsive disorder. Journal of Consulting and Clinical Psychology, 69, 205-214. 
Mortiz, S., Rufer, M., Fricke, S., Karow, A., Morfeld, M., Jelinek, L., \& Jacobsen, D. (2005). Quality of life in obsessive-compulsive disorder before and after treatment. Comprehensive Psychiatry, 46, 453-359.

Norberg, M.M., Calamari, J.E., Cohen, R.J., \& Riemann, B.C. (2008). Quality of life in obsessive-compulsive disorder: An evaluation of impairment and a preliminary analysis of the ameliorating effects of treatment. Depression and Anxiety, 25, 248-259.

Pallanti, S., Hollander, E., Bienstock, C., Koran, L., Leckman, J., Marazziti, D., Pato, M., Stein, D., Zohar, J., \& the International Treatment Refractory OCD Consortium. (2002). Treatment non-response in OCD: Methodological issues and operational definitions. International Journal of Neuropsychopharmacology, 5, 181-191.

Rosa-Alcázar, A.I., Sánchez-Meca, J., Gómez-Conesa, A., \& Marín-Martínez, F. (2008). Psychological treatment of obsessive-compulsive disorder: A metaanalysis. Clinical Psychology Review, 28, 1310-1325.

Sanavio, S. (1988). Obsessions and compulsions: The Padua Inventory. Behaviour Research and Therapy, 26, 169-177.

Somers, J.M., Goldner, E.M., Waraich, P., \& Hsu, L. (2006). Prevalence and incidence studies of anxiety disorders: A systematic review of the literature. Canadian Journal of Psychiatry, 51, 100-113.

Stewart, S.E., Stack, D.E., Farrell, C., Pauls, D.L., \& Jenike, M.A. (2005). Effectiveness of intensive residential treatment (IRT) for severe, refractory obsessive-compulsive disorder. Journal of Psychiatric Research, 39, 603-609. 
Stewart, S.E., Yen, C-H., Stack, D.E., \& Jenike, M.A. (2006). Outcome predictors for severe obsessive-compulsive patients in intensive residential treatment. Journal of Psychiatric Research, 40, 511-519.

Thornicroft, G., Colson, L. \& Marks, I. (1991). An in-patient behavioural psychotherapy unit: Description and audit. British Journal of Psychiatry, 158, $362-367$.

van Balkom, A.J.L.M, van Oppen, P., Vermeulen, A.W.A., van Dyck, R., Nauta, M.C.E., \& Vorst, H.C.M. (1994). A meta-analysis on the treatment of obsessive compulsive disorder: A comparison of antidepressants, behavior, and cognitive therapy. Clinical Psychology Review, 14, 359-381.

van Oppen, P., De Haan, E., Van Balkom, A.J.L.M., Spinhoven, P., Hoogduin, K., \& van Dyck, R. (1995). Cognitive therapy and exposure in vivo in the treatment of obsessive compulsive disorder. Behaviour Research and Therapy, 33, 379390. 


\section{Footnote}

${ }^{1}$ One reviewer questioned whether the high level of depression symptoms in the sample also reflected a skewed distribution or a distribution with little variance that may have distorted this finding. We were able to rule this out through examination of the descriptive statistics of the baseline BDI measurement which showed a normal distribution, with adequate variance. 
Table 1

Previous Treatment Experience of the Cohort

\begin{tabular}{ll}
\hline Previous Treatment & Percentage
\end{tabular}

Exposure with Response Prevention ( $\geq 1$ Trial) $\quad 83.0 \%$

Exposure with Response Prevention ( $\geq 2$ Trials) $\quad 37.9 \%$

$\begin{array}{ll}\text { Cognitive Behaviour Therapy* } & 85.7 \%\end{array}$

Serotonin Reuptake Inhibitors ( $\geq 1$ Trial) $\quad 76.0 \%$

Serotonin Reuptake Inhibitors ( $\geq 2$ Trials) $\quad 40.0 \%$

Augmentation with Dopamine Antagonist $\quad 16.5 \%$

* Not necessarily including exposure with response prevention 
Table 2

Patient Characteristics

Demographic Variable

Number (Percentage)

Marital Status

Single

$117(57.1 \%)$

Married / Cohabiting

$53(25.9 \%)$

Divorced / Separated

$4(2.0 \%)$

Unrecorded / Missing

$31(15.1 \%)$

Employment Status

Unemployed

$78(38.0 \%)$

Full-Time Employment

$84(41.0 \%)$

Part-Time or Voluntary Employment

$18(8.8 \%)$

Houseperson

$5(2.4 \%)$

Student

$4(2.0 \%)$

Unrecorded / Missing

$16(7.8 \%)$

Accommodation Status

Living Alone

$34(16.6 \%)$

Living with Parents

$26(12.7 \%)$

Living with Partner or Spouse

$47(22.09 \%)$

Living in Supported Accommodation

$3(1.5 \%)$

Unrecorded / Missing

$95(46.3 \%)$ 
Table 3

Additional Comorbid Diagnoses

\begin{tabular}{ll}
\hline Additional Comorbid Diagnosis & Number (Percentage) \\
\hline Any Additional Diagnosis & $99(48.3)$ \\
Body Dysmorphic Disorder & $7(3.4 \%)$ \\
Major Depressive Disorder & $68(33.2 \%)$ \\
Schizophrenia & $2(1.0 \%)$ \\
Eating Disorder & $4(2.0 \%)$ \\
Personality Disorder & $2(1.0 \%)$ \\
Posttraumatic Stress Disorder & $4(2.0 \%)$ \\
Substance Use Disorder & $9(4.4 \%)$ \\
Other Anxiety Disorder & $48(23.4 \%)$ \\
\hline
\end{tabular}




\section{Figure Captions}

Figure 1. Flow of referred patients through treatment.

Figure 2. Change in total Y-BOCS and BDI scores during treatment.

Figure 3. Change in total Padua Inventory scores during treatment.

Figure 4. Benchmarking of current results against previous research.

Figure 5. Comparison of proportion of patients who improved or recovered with

Fisher \& Wells (2005) and Boschen et al. (2008). 


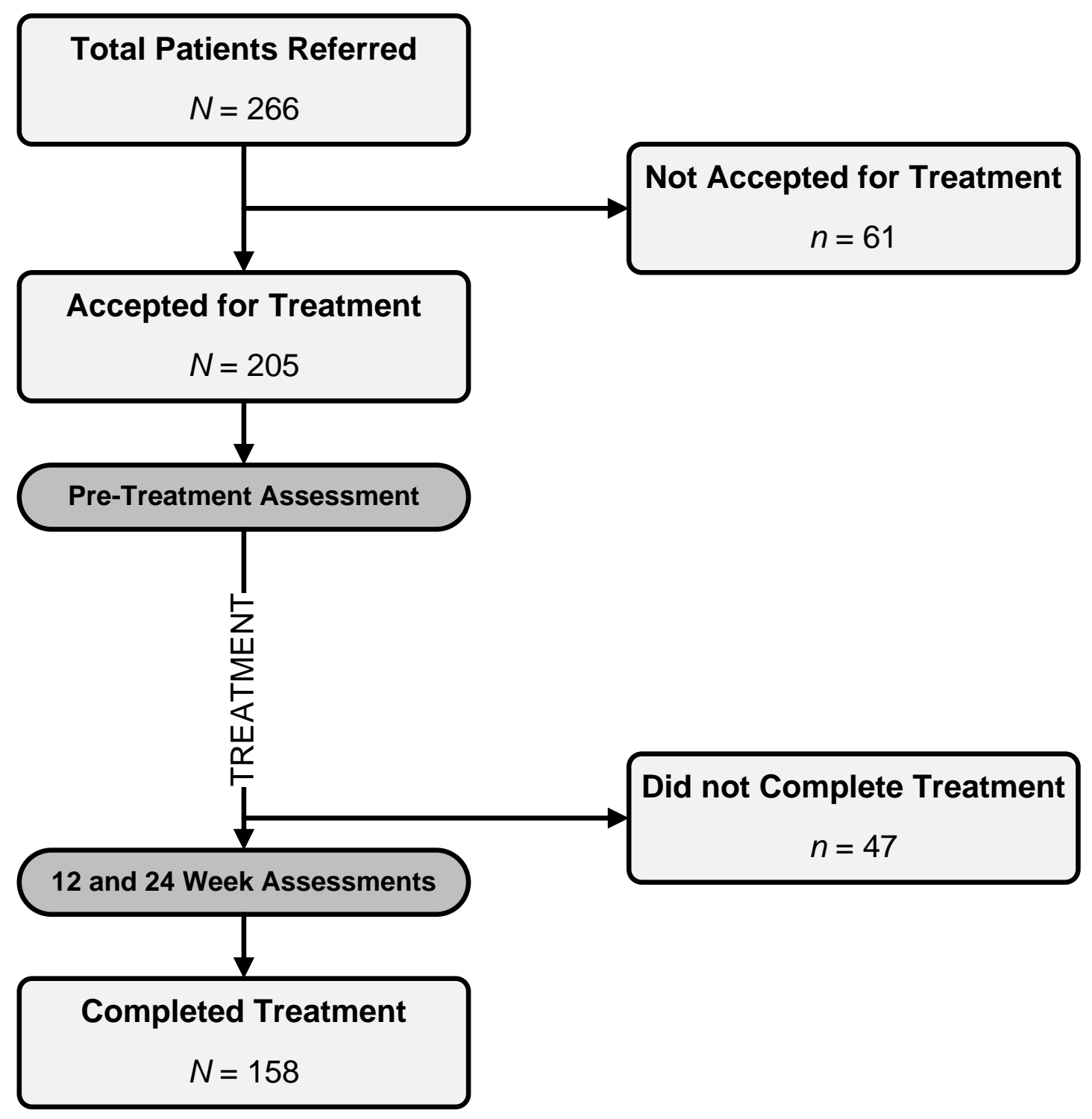




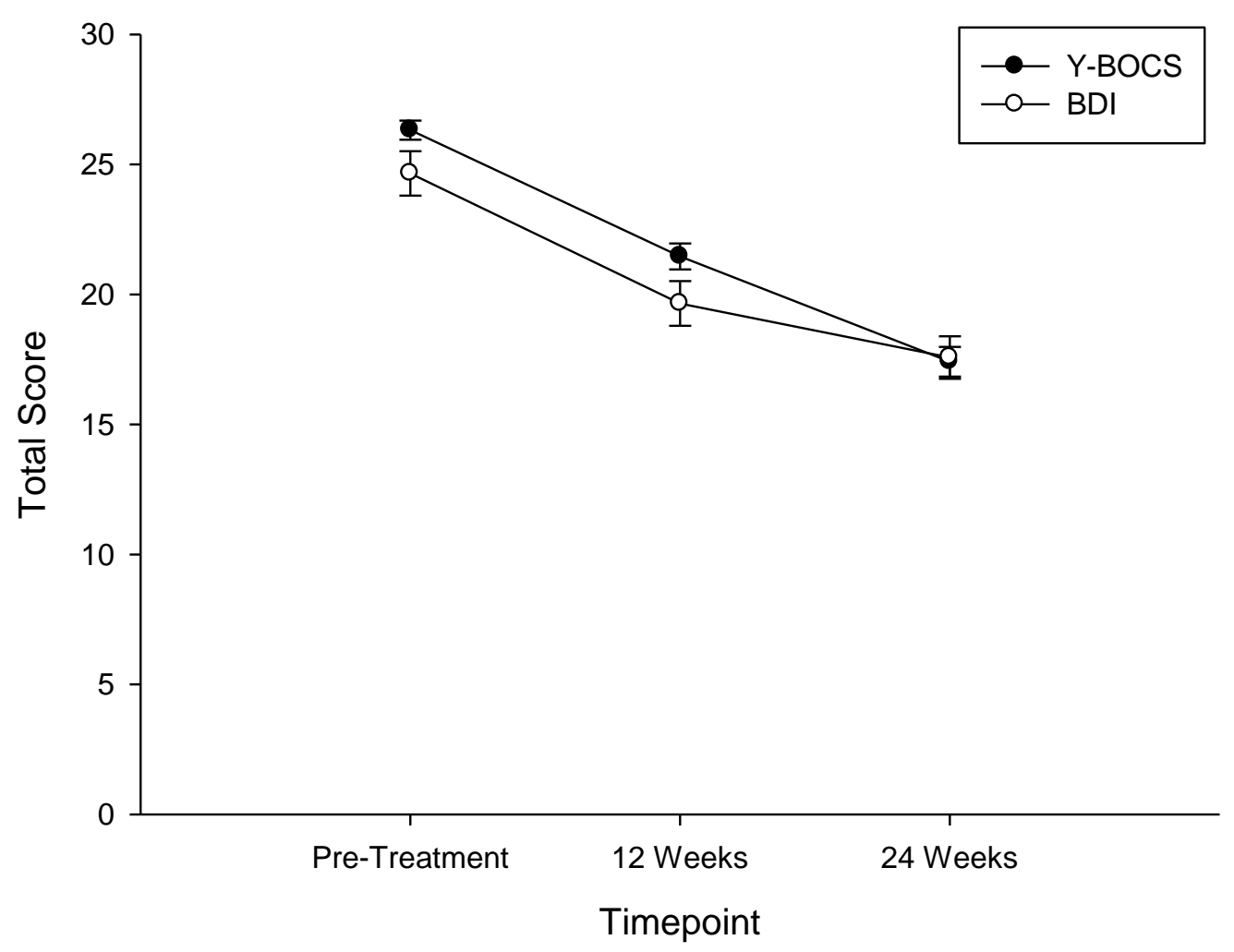




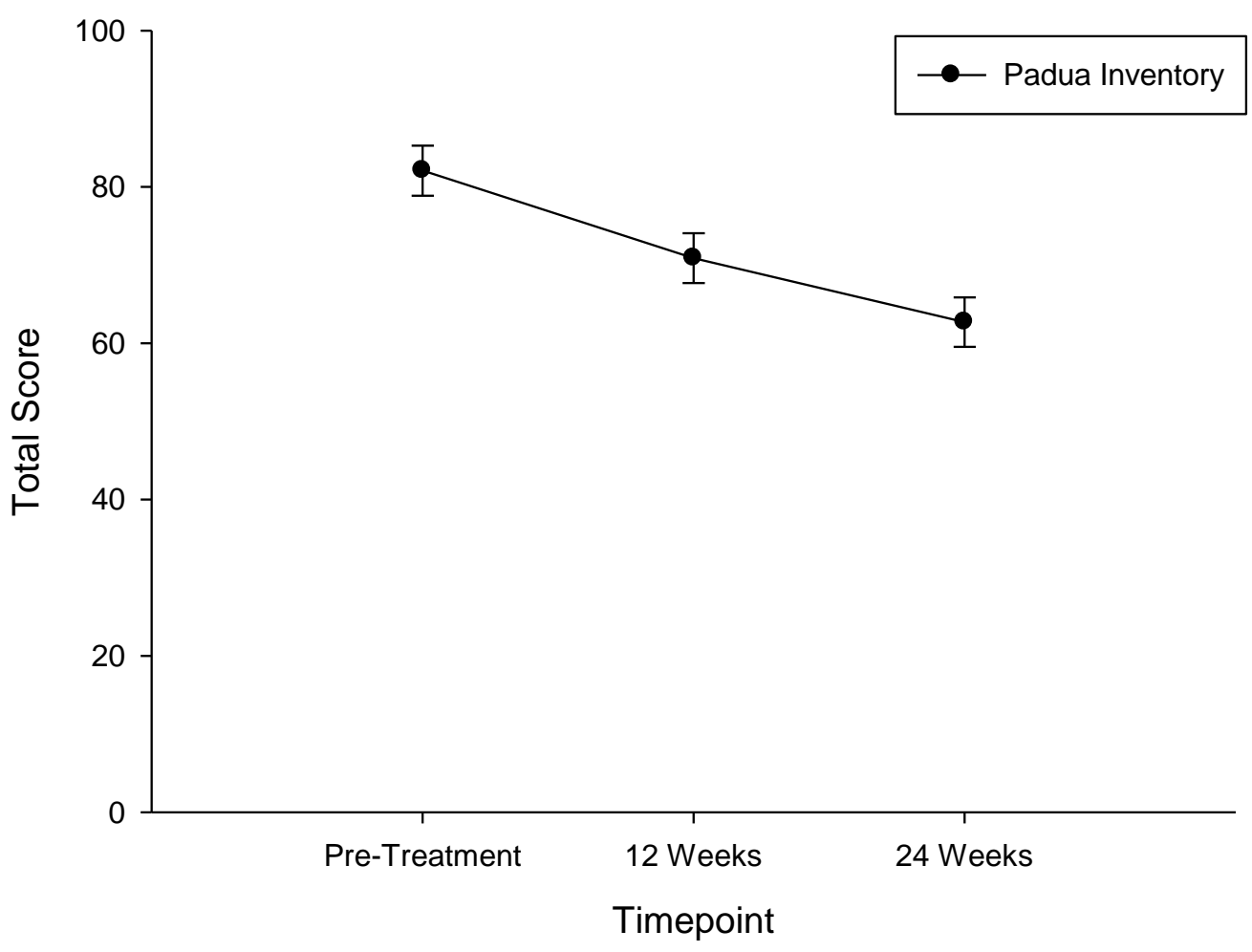




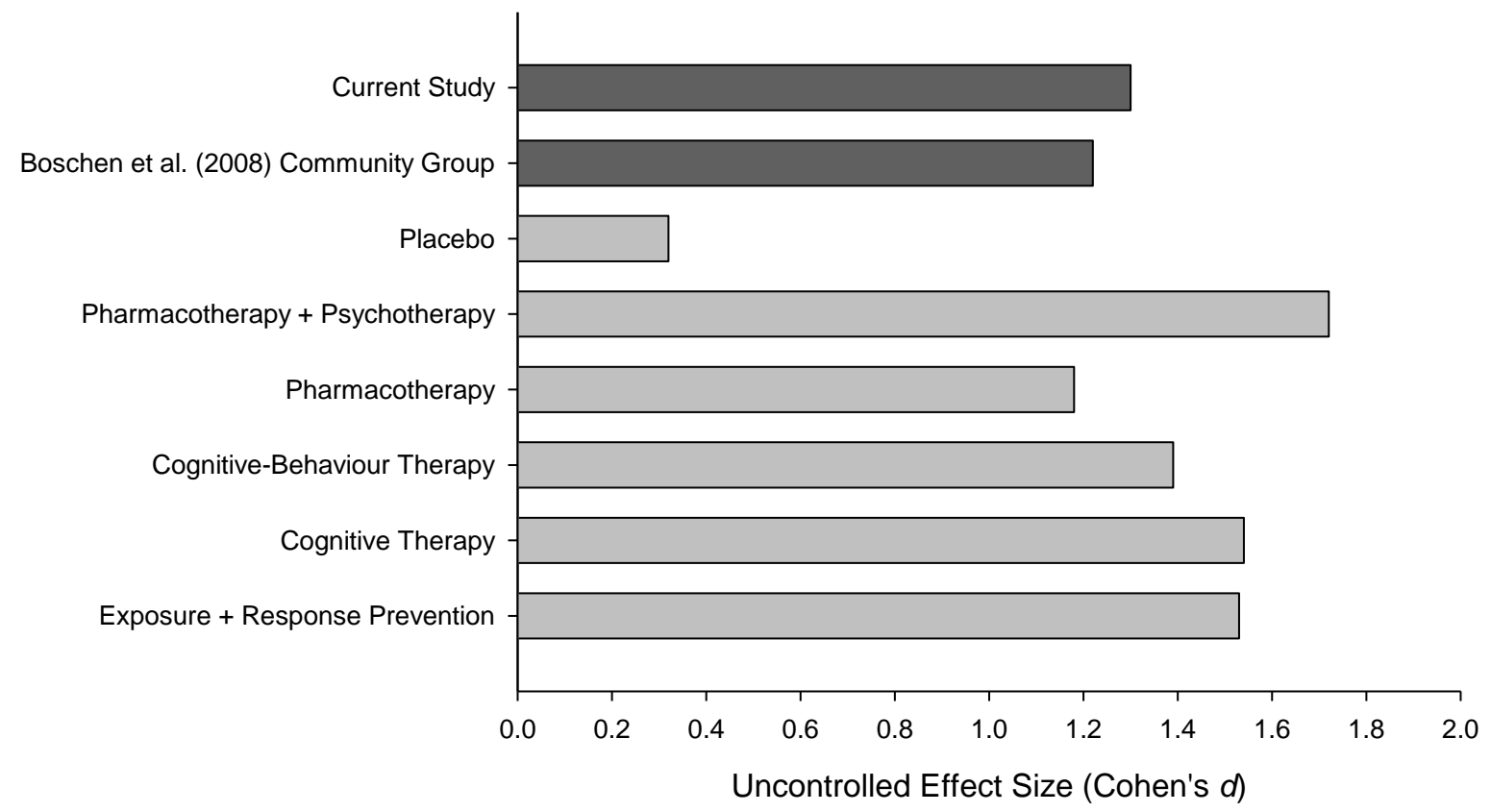




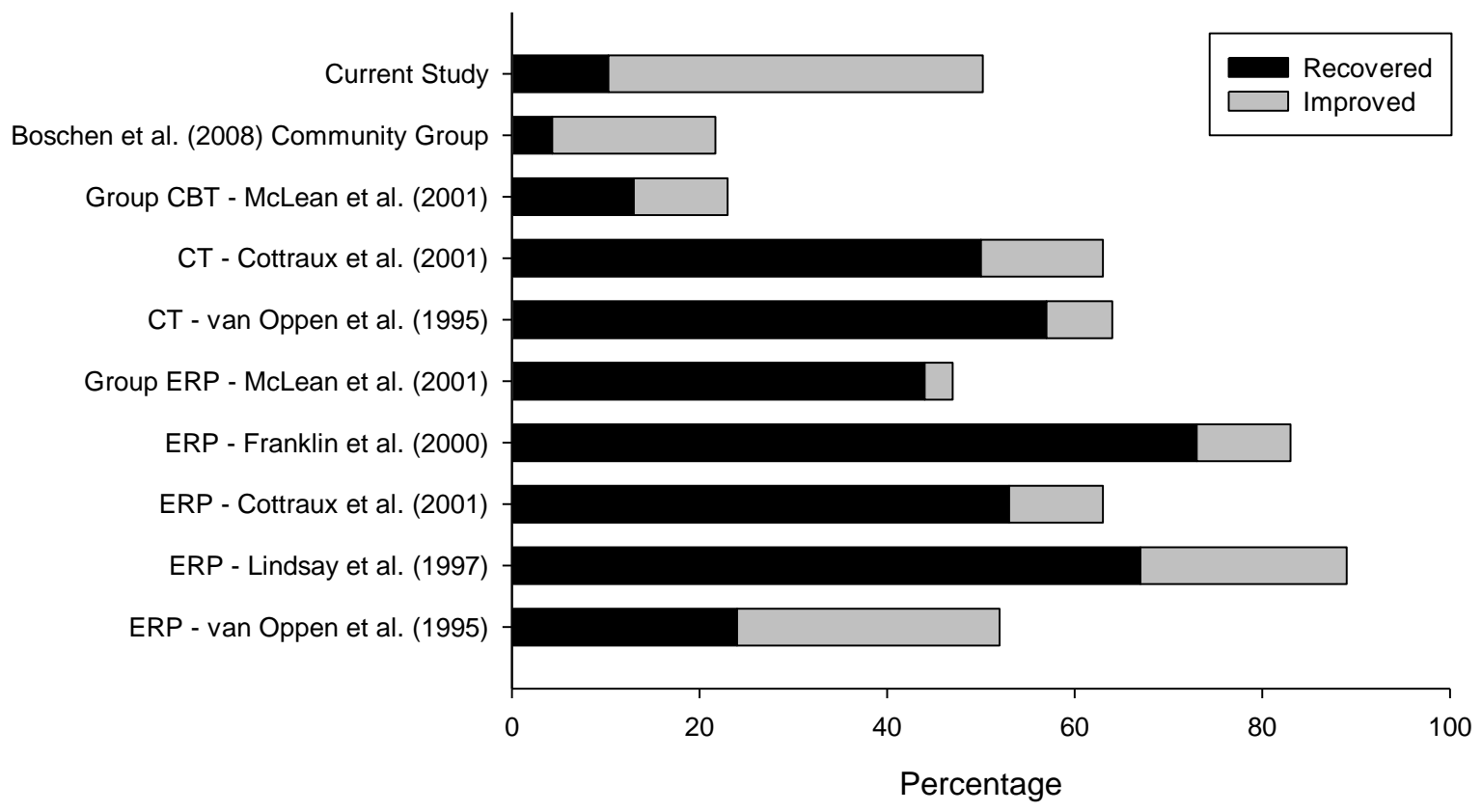

